\title{
Chronic ethanol intake leads to structural and molecular alterations in the rat endometrium
}

\author{
Marcelo Martinez ${ }^{a}$, Flora A. Milton ${ }^{\mathrm{a}, 1}$, Patricia Fernanda F. Pinheiro ${ }^{\mathrm{b}, 1}$, Camila C.D. Almeida-Francia ${ }^{\mathrm{b}}$, \\ Valeria H.A. Cagnon-Quitete ${ }^{\mathrm{d}}$, Luiz F. Tirapelli ${ }^{\mathrm{e}}$, Carlos Roberto Padovani ${ }^{\mathrm{c}}$, Luiz Gustavo A. Chuffa ${ }^{\mathrm{b}}$, \\ Francisco Eduardo Martinez ${ }^{\mathrm{b}, *}$
}

${ }^{a}$ Department of Morphology and Pathology, UFSCar, São Carlos, SP, Brazil

${ }^{\mathrm{b}}$ Department of Anatomy, UNESP, Botucatu, SP, Brazil

${ }^{\mathrm{c}}$ Department of Biostatistics, UNESP, Botucatu, SP, Brazil

d Department of Anatomy, Cellular Biology, Physiology and Biophysics, UNICAMP, Campinas, SP, Brazil

e Department of Surgery and Anatomy, USP, Ribeirão Preto, SP, Brazil

\section{A R T I C L E I N F O}

\section{Article history:}

Received 4 March 2015

Received in revised form

10 December 2015

Accepted 3 February 2016

\section{Keywords:}

Uterus

Epithelium

Apoptosis

TEM

Ethanol

UCh rat

\begin{abstract}
A B S T R A C T
We described the effects of low- and high-dose ethanol intake on the structure and apoptosis signaling of the uterine endometrium of UChA and UChB rats (animals with voluntary ethanol consumption). Thirty adult female rats, 90 days old, were divided into three groups ( $n=10$ /group): UChA rats fed with $10 \%(\mathrm{v} /$ v) ethanol ad libitum (free choice for water or ethanol) drinking $<1.9 \mathrm{~g} / \mathrm{kg} /$ day; UChB rats fed with $10 \%$ $(\mathrm{v} / \mathrm{v})$ ethanol ad libitum (free choice for water or ethanol) drinking from 2 to $5 \mathrm{~g} / \mathrm{kg} /$ day; control rats without ethanol (only water). After 120 days of treatment, rats displaying estrus were euthanized. Uterine epithelial cells of the UCh rats showed dilated cisterns of the rough endoplasmic reticulum, presence of lipid droplets, altered nuclear chromatin, and disrupted mitochondria. The UCh rats exhibited intense atrophied epithelial cells with smaller areas and perimeters of cytoplasm and nuclei. The endometrium of UChA rats showed higher levels of caspase- 3 while Xiap and Bcl2 varied from moderate to weak. Both UChA and UChB rats exhibited a stronger immunoreaction to Ki-67 and IGFR-1 on epithelial and stromal cells. Chronic ethanol intake leads to structural and molecular alterations in the uterine endometrium of UCh rats, regardless of low- or high-dose consumption, promoting reproductive disorders.
\end{abstract}

(c) 2016 Elsevier Inc. All rights reserved.

\section{Introduction}

Ethanol ingestion has increased in recent decades with social consequences and has become one of the most serious problems of global public health (World Health Organization, 2011). It is a toxic agent that disturbs the integrity of biochemical and physiological functions and the development of organic structures involved in reproduction, causing severe damage to the signaling of hypothalamic-pituitary-gonadal (HPG) and hypothalamicpituitary-adrenal (HPA) axes (Wallock-Montelius et al., 2007). Ethanol may affect the pregnancy rate with higher risk of spontaneous abortions; prolonged consumption can lead to male and female infertility (Clave, Joya, Salat-Batlle, Garcia-Algar, \& Vall, 2014; Kesmodel, Wisborg, Olsen, Henriksen, \& Secher, 2002).

\footnotetext{
* Corresponding author. Tel.: +55 143880 0024; fax: +55 1438153744 .

E-mail address: martinez@ibb.unesp.br (F.E. Martinez).

1 These authors contributed equally to the paper.
}

Furthermore, chronic ethanol exposure is associated with malformations in fetal development (Ornoy \& Ergaz, 2010) and some cancers (Surtel, Klepacz, \& Wysokińska-Miszczuk, 2014).

The prevalence of ethanol abuse and dependence is higher among men. However, recent studies have shown that consumption among women is growing, while male consumption has remained stable (Day, Helsel, Sonon, \& Goldschmidt, 2013; Lyngsø et al., 2014). Researchers showed that women initiate ethanol consumption later than men, but seek treatment at the same age, when they develop an alcohol-associated pathology, which can be associated with more rapid progression of alcoholism in women with morbidity varying from 1.5 to 2 times higher compared to men with a similar pattern of intake (Fernandez-Sola, Nicolas, Estruch, \& UrbanoMarquez, 2005; Hochgraf, Zilberman, \& Andrade, 1995). Only a few consequences of excessive ethanol ingestion on uterine tissue have been reported in the literature, such as atrophy of the uterine horn and endometrial lining thickness, and morphometrical and ultrastructural alterations to the epithelial and glandular cells 
(Buthet et al., 2013; Martinez et al., 1999, 2001). Recent findings have focused on the effects of chronic ethanol consumption and the relationship between circulating sex hormones and their receptors in female reproduction (Chuffa et al., 2013). Specifically to uterine tissue, long-term ethanol consumption was negatively correlated to the levels of androgen receptor (AR), progesterone receptor (PR), and estrogen receptor (subunit ER- $\alpha$ ).

According to Buthet et al. (2013), the rat uterine horn has different metabolic pathways able to generate acetaldehyde and free radicals from ethanol. This long-term accumulation may cause cell injury, and promote cancer and related pathologies. We have previously described that high ethanol consumption by rats is associated with induction of apoptosis in different tissues (Oliveira, Chuffa, et al., 2014; Oliveria, Fontanelli, et al., 2014), which may occur due to oxidative stress (Chu, Tong, \& Monte, 2007). While most studies have reported that tissue damage arises as a result of prenatal alcohol exposure, we proposed to evaluate the function of endometrial lining during the estrus phase, focusing on apoptotic and anti-apoptotic signaling. To date, no study has addressed the effects of high- and low-ethanol intake on uterine apoptosis.

The UCh ethanol-preferring rat model (Mardones \& SegoviaRiquelme, 1983) is derived from original Wistar rats and represents a special model for understanding the basis of alcoholismlinked characteristics such as those described in alcohol-related human diseases. Therefore, the present study aimed to investigate the effects of low- and high-dose ethanol consumption on the structure and apoptotic signaling of the uterine endometrium in two ethanol-preferring rat models.

\section{Materials and methods}

\section{Animals and experimental design}

Thirty adult female rats (Rattus norvegicus albinus), 90 days old, were obtained from the Department of Anatomy, Bioscience Institute/Campus of Botucatu (IBB/UNESP) - Univ Estadual Paulista. The rats were divided into three groups ( $n=10$ /group): UChA rats fed with 1:10 (v/v) ethanol ad libitum (free choice for water or ethanol) drinking from 0 to $1.9 \mathrm{~g} / \mathrm{kg} /$ day; UChB rats fed with $1: 10(\mathrm{v} / \mathrm{v})$ ethanol ad libitum (free choice for water or ethanol) drinking from 2.0 to $6.0 \mathrm{~g} / \mathrm{kg} /$ day; control rats without access to ethanol (only water). When the animals reached 60 days of age they were given, during a period of 20 days, a choice between two bottles containing either water ad libitum (1) or $10 \%(v / v)$ ethanol solution (2). Afterward, 10 animals per group displaying ethanol consumption within the expected average were selected according to the criteria of Mardones and Segovia-Riquelme (1983). In this study, the preference ratio associated with ethanol-seeking behavior was approximately $60 \%$. To ensure higher efficiency and to maintain constant consumption throughout the experiment, rats were kept under observation for 10 days. During all the treatments, estrus cycles were monitored daily at 9:00 AM using a vaginal-swab technique. All rats were housed in polypropylene cages $(43 \times 30 \times 15 \mathrm{~cm})$ with laboratory-grade pine shavings as bedding and maintained under controlled temperature $\left(23 \pm 1^{\circ} \mathrm{C}\right)$ and lighting conditions $(12 \mathrm{~h}$ light/12 h dark photoperiod, lights switched off at 7:00 AM). Experimental protocols followed the Ethical Principles in Animal Research of the Brazilian College of Animal Experimentation (no65/ 07-CEEA). After 120 days of treatment, females in estrus phase were anesthetized and euthanized by decapitation for further analysis.

\section{Body, genital organs weight, and tissue processing}

The rats were weighed in an Owalabor analytical balance and submitted to abdominal-pelvic laparotomy in order to collect genital organs (uterine horn, ovaries, and uterine tubes), which were weighed together. The uterine horn, ovaries, and uterine tubes were dissected under a surgical microscope (D. F. Vasconcelos), and the uterus fixed in Bouin solution during $6 \mathrm{~h}$. After sequential washes, the uterus was conditioned in $70 \%(v / v)$ ethanol. Dehydration steps were made using $80 \%, 90 \%, 95 \%$, and $100 \%$ alcohol for $4 \mathrm{~h}$, following diaphanization and paraplast embedding (Oxford Labware, St. Louis, MO, USA).

\section{Morphometry, light microscopy, and immunohistochemistry}

The blocks were sectioned at $4 \mu \mathrm{m}$ thickness using a LEICA 2145 microtome and stained with hematoxylin and eosin (H\&E). The slides were imaged by digital photomicroscope (Axiophot II Zeiss). For histological analysis and counting measures, slides with H\&E were used, and the values were obtained in five slides per rat in three areas per section with $20 \times$ magnification (interval between each section was $50 \mu \mathrm{m}$ ).

Rabbit primary antibodies (1:100 Santa Cruz Biotechnology, CA, USA) were used for IGFR-1, Ki-67, Bcl2, and Xiap analysis, and rabbit primary antibodies (1:100 Biocare, USA) were used for caspace-3 analysis, according to the manufacturers' procedures $(n=5 /$ group). After immunoreactions, the slides were washed in TBS-T buffer and incubated with secondary antibody (anti-mouse IgG or anti-rabbit IgG; DAKO ${ }^{\circledR}$ CYT; Glostrup, Denmark) for $1 \mathrm{~h}$. Then, slides were reacted with diaminobenzidine (DAB; Sigma, St. Louis, MO, USA) and counterstained with hematoxylin. Five slides of each uterine horn containing four sections were stained for morphometric analysis. The immunohistochemistry results were analyzed by a pathologist using a Zeiss Axiophot II microscope (Carl Zeiss, Oberkochen, Germany), based on the levels of staining intensity, which were scored as absent $(0)$, weak $(+)$, moderate $(++)$, or strong $(+++/++++)$ immunoreactivity.

\section{Transmission Electron Microscopy (TEM)}

For TEM analysis, five rats per group were decapitated, and the uterine horn was collected and immersed in modified Karnovsky's fixative (2.5\% glutaraldehyde, $2 \%$ paraformaldehyde, $0.1 \mathrm{M}$ sodium phosphate buffer, $\mathrm{pH} 7.2$ ). The $0.5 \mathrm{~mm}$ uterine horn fragments remained in the fixative solution for $3 \mathrm{~h}$ at $4{ }^{\circ} \mathrm{C}$. Afterward, they were post-fixed in $1 \%$ osmium tetroxide $\left(\mathrm{OsO}_{4}\right)$, sodium phosphate buffer, and then embedded in Araldite ${ }^{\circledR}$ plastic resin. After embedding, sections $0.5 \mu \mathrm{m}$ thick were obtained by using an LKB 8800 ultramicrotome, Ultratome III, with a glass blade, and stained with toluidine blue and azur II. After selecting specific areas, the blocks were trimmed and ultrathin sections were obtained, being contrasted by uranyl acetate and lead citrate. All the analysis and documentation were performed using a Philips CM 100 transmission electron microscope (Electron Microscopy Center of IBB/UNESP).

\section{Scanning Electron Microscopy (SEM)}

The uterine horns were fixed with Karnovsky's solution for $2 \mathrm{~h}$ and post-fixed with $1 \% \mathrm{OsO}_{4}$ for $2 \mathrm{~h}$. They were immersed in $2 \%$ tannic acid for $2 \mathrm{~h}$, dehydrated in graded ethanol, which was replaced with isoamyl acetate, and dried to a critical point with $\mathrm{CO}_{2}$ (Balzers CPD-010). The specimens were coated with gold (Balzers MED-010) and examined in a Philips FEM 515 scanning electron microscope.

\section{Hormone levels}

After decapitation, trunk blood samples were obtained from rats ( $n=10$ /group) to verify LH, FSH, estradiol, and progesterone, and 
Table 1

Body weight, genital organs weight, and morphometrical data of uterine horn of ethanol-preferring rats.

\begin{tabular}{|c|c|c|c|}
\hline Parameters & UChA & UChB & Control \\
\hline${ }^{\mathrm{a} B o d y}$ weight (g) & $221.00 \pm 17.88 \mathrm{a}$ & $253.00 \pm 17.88 b$ & $232.67 \pm 12.58 \mathrm{ab}$ \\
\hline $\begin{array}{c}{ }^{\mathrm{a}} \text { Genital organs } \\
\text { weight }(\mathrm{g})\end{array}$ & $1.11 \pm 0.20 \mathrm{a}$ & $0.94 \pm 0.25 \mathrm{a}$ & $1.24 \pm 0.31 \mathrm{a}$ \\
\hline $\begin{array}{l}\text { aterine epithelial } \\
\text { height }(\mu \mathrm{m})\end{array}$ & $43.88 \pm 8.27 \mathrm{a}$ & $85.89 \pm 12.82 b$ & $98.48 \pm 23.26 b$ \\
\hline $\begin{array}{l}\text { Uterine epithelial } \\
\text { cyto area }\left(\mu \mathrm{m}^{2}\right)\end{array}$ & $56.54 \pm 6.34 \mathrm{a}$ & $107.87 \pm 30.21 b$ & $243.66 \pm 67.70 \mathrm{c}$ \\
\hline $\begin{array}{l}{ }^{\mathrm{b}} \text { Uterine epithelial } \\
\text { cyto perimeter } \\
(\mu \mathrm{m})\end{array}$ & $31.07 \pm 1.14 \mathrm{a}$ & $49.08 \pm 6.79 b$ & $80.47 \pm 19.22 \mathrm{c}$ \\
\hline $\begin{array}{l}\text { buterine epithelial } \\
\text { nucleus area }\left(\mu \mathrm{m}^{2}\right)\end{array}$ & $13.66 \pm 1.13 \mathrm{a}$ & $21.44 \pm 7.18 b$ & $63.06 \pm 11.48 c$ \\
\hline $\begin{array}{l}{ }^{\mathrm{b}} \text { Uterine epithelial } \\
\text { nucleus } \\
\text { perimeter }(\mu \mathrm{m})\end{array}$ & $14.07 \pm 0.59 a$ & $18.11 \pm 2.22 b$ & $30.95 \pm 3.33 c$ \\
\hline $\begin{array}{l}\text { buterine } \\
\quad \text { diameter }(\mu \mathrm{m})\end{array}$ & $2872.5 \pm 724.9 \mathrm{a}$ & $2104.4 \pm 358.6 \mathrm{a}$ & $2538.4 \pm 575.8 a$ \\
\hline
\end{tabular}

Means followed by different letter differ statistically among the groups $(p<0.05)$.

a One-way ANOVA complemented by Tukey's test (values expressed as mean \pm SD)

b For non-parametric measures, Kruskal-Wallis test complemented by Dunn was applied (median \pm semi-amplitude); $n=10$ /group.

the plasma concentration was achieved by double antibody radioimmunoassay method (RIA). Specific antibodies anti-rat LH-S10 and FSH-S11 were diluted in rabbit serum. The second antibody was isolated from sheep using rabbit anti-gammaglobulin. All samples were run in the same assay for each hormone to avoid interassay variations.

\section{Statistics}

The values were presented as the mean \pm S.D., and data analyses were performed using one-way ANOVA. Significant results were subjected to post hoc testing using Tukey's test, and statistical significance was set at $p<0.05$ (Banzatto \& Kronka, 1989).

\section{Results}

\section{Morphometric data and hormones}

The UChB rats had higher body weight, whereas no significant difference was observed in reproductive organ weight. Despite the fact that only UChA rats showed a decrease in the uterine epithelial height, both UChA and UChB rats were affected by the ethanol, displaying severe reduction in the area and perimeter of cytoplasm and nuclei of uterine epithelial cells (Table 1). No changes in plasma FSH, $\mathrm{LH}$, progesterone, and $17 \beta$-estradiol were found in UCh rats (Table 2 ).

\section{Histological analysis}

The endometrium of UChA rats appeared to be reduced in thickness compared to UChB and control animals (Fig. 1). The

Table 2

Plasma hormone levels in rats.

\begin{tabular}{lccc}
\hline \multirow{2}{*}{ Parameters } & \multicolumn{3}{c}{ Groups } \\
\cline { 2 - 4 } & UChA & UChB & Control \\
\hline FSH $(\mathrm{mIU} / \mathrm{mL})$ & $6.5 \pm 3.85 \mathrm{a}$ & $3.90 \pm 2.85 \mathrm{a}$ & $5.25 \pm 2.85 \mathrm{a}$ \\
LH $(\mathrm{mIU} / \mathrm{mL})$ & $0.40 \pm 0.85 \mathrm{a}$ & $0.20 \pm 1.15 \mathrm{a}$ & $0.70 \pm 4.50 \mathrm{a}$ \\
Progesterone $(\mathrm{ng} / \mathrm{mL})$ & $19.34 \pm 13.28 \mathrm{a}$ & $30.25 \pm 12.97 \mathrm{a}$ & $22.16 \pm 12.79 \mathrm{a}$ \\
$17 \beta$-estradiol $(\mathrm{pg} / \mathrm{mL})$ & $47.66 \pm 37.90 \mathrm{a}$ & $44.73 \pm 39.29 \mathrm{a}$ & $43.57 \pm 19.22 \mathrm{a}$ \\
\hline
\end{tabular}

Means followed by different letter differ statistically among the groups $(p<0.05)$. Kruskal-Wallis test complemented by Dunn (values expressed as median \pm semiamplitude; $n=10$ /group). uterine lining of UChA rats showed an intense atrophy displayed by cuboidal cells, and the stroma was edematous with variations in collagen-fiber spacing (Fig. 1B). Conversely, the epithelium of UChB rats was high columnar with nuclei located at different levels and no mitotic activity. The cellular stroma was common in UChB rats (Fig. 1C). Similar to control animals, vacuolated epithelial cells containing fragments of degenerating chromatin were notable in the epithelium of UChB rats, suggesting intense activity by these cells (Fig. 1A and C).

\section{Immunohistochemistry for apoptotic and anti-apoptotic proteins}

The UChB rats showed a weak immunoreaction to caspase-3, $\mathrm{Bcl} 2$, and Xiap in the endometrium that was similar to control animals (Fig. 2A, C, D, F, G, I; Table 3). Only UChA rats showed an intense positive reaction to caspase- 3 whereas a moderate to weak reaction was observed to $\mathrm{Bcl} 2$ and Xiap (Fig. 2B, E, H; Table 3). Both UChA and $U C h B$ rats presented a stronger immunoreaction to Ki-67 and IGFR-1 (Fig. 2J-O; Table 3), suggesting a continuous proliferative activity in epithelial and stromal cells of the endometrium.

\section{TEM and SEM analysis}

The endometrial lining of UChA rats showed an intense atrophy. In UChB rats, the cisterns of the rough endoplasmic reticulum showed marked dilation compared to the control group (Fig. 3A and B). The epithelial cells of the UChA and UChB rats showed lipid droplets of different sizes throughout the cytoplasm, either clustered together or associated with mitochondria and vacuoles (Fig. 3C and D). Furthermore, these vacuoles containing the engulfed material varied in sizes and shapes regarding different regions of the cytoplasm (Fig. 3D). Hypertrophied and elongated mitochondria with voluminous matrices and cristae were only found in the UChA rats (Fig. 3E). In these ethanol-preferring rats, nuclei with condensed peripheral chromatin as well as chambers and deformities caused by the presence of large vacuoles were common (Fig. 3F). The luminal surface of the endometrium of UChA and UChB rats showed increased intercellular limits. This fractured material revealed the presence of lipid droplets in the uterine cavity of the UCh rats (Fig. 3G-I).

\section{Discussion}

Body weight of UChB rats was significantly higher compared to the UChA rats, as has been already proposed by Martinez et al. (1999, 2001). Grinfeld, Goldenberg, Segre, and Chadi (1999) and Buthet et al. (2013) found no significant differences between the body weights of mice and pregnant rats treated with liquid diet containing $27.5 \%$ of the calories derived from ethanol. Moreover, Bo, Kruegen, Rudeen, and Symmes (1982) showed that the average body weight gain in Holtzman rats treated with ethanol at $2.5 \%$ and $5 \%$ during 28-77 days of age was lower in those receiving 5\% ethanol. Our study may reflect the use of ethanol-derived calories which could not have prevent weight loss, often associated with chronic alcoholism, as it produced the same weight gain observed in control rats. Furthermore, Martinez et al. (1999, 2001) have reported lethargy in these UChB rats, with reduced energy expenditure and weight loss.

The height of the UChA epithelium was significantly lower compared to controls. These results are in agreement with Martinez et al. (1999, 2001) who reported atrophy in the uterine epithelium of rats intoxicated by ethanol at different endpoints and treatments $(90,180$, and 270 days). Martinez et al. (1999) also observed a significant difference in the cytoplasm and nuclei of epithelial cells between the groups at different periods. The uterine epithelium of 

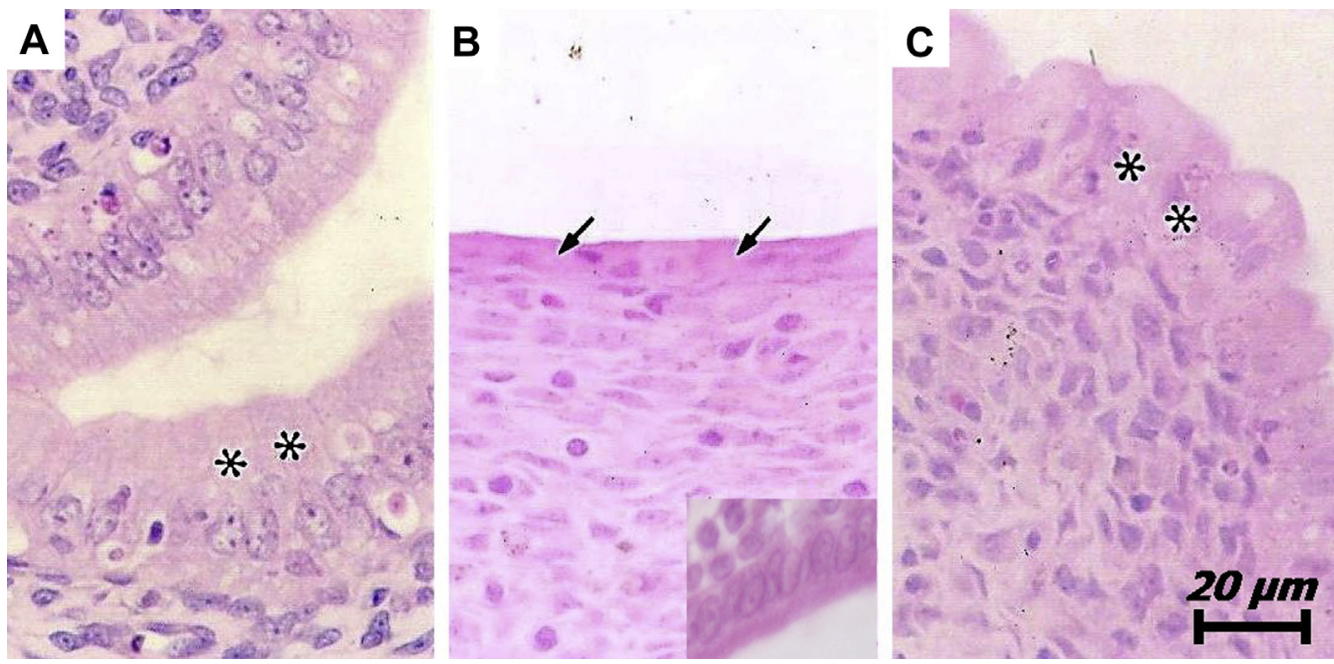

Fig. 1. Uterine horn of Control (A), UChA (B), and UChB (C) rats. Note the columnar epithelium $\left.{ }^{* *}\right)$ typical of endometrial lining and intense atrophy in the epithelial layer of the UChA group (arrows). H\&E staining. Bar $=20 \mu \mathrm{m}$. For detail, bar $=10 \mu \mathrm{m}$.

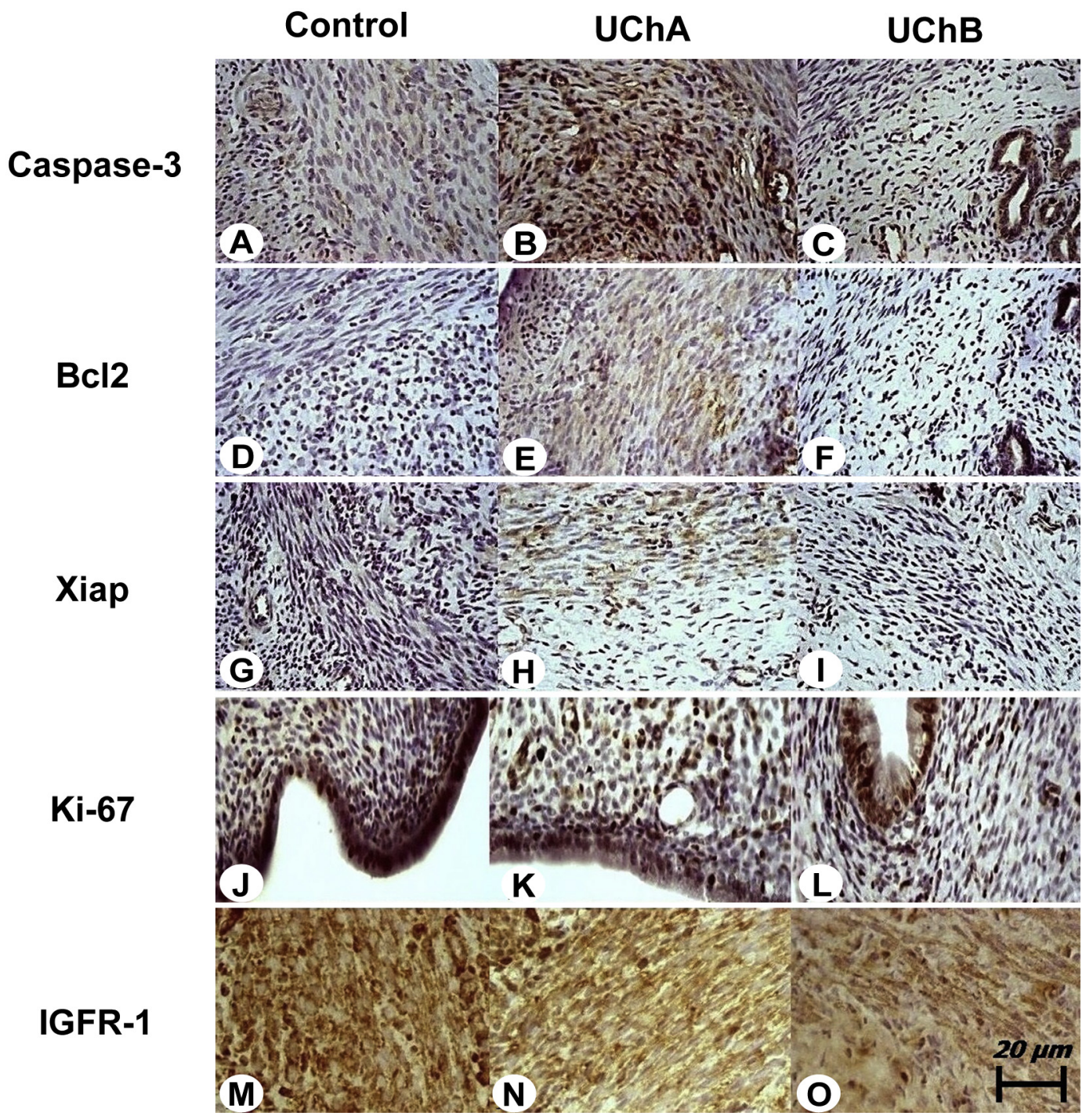

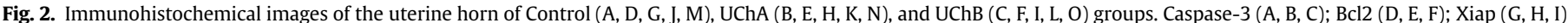

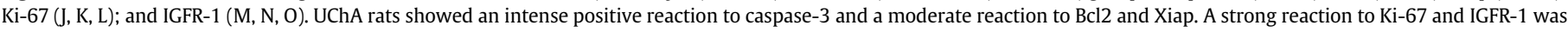
observed in the UCh rats. $\mathrm{Bar}=20 \mu \mathrm{m}$. 
Table 3

Intensity of immunoreactivity of caspase-3, Bcl2, Xiap, Ki-67, and IGFR-1 in the uterine horn of rats.

\begin{tabular}{llll}
\hline Antigen & Control & UChA & UChB \\
\hline Caspase-3 & + & +++ & + \\
Bcl2 & + & ++ & + \\
Xiap & + & ++ & + \\
Ki-67 & ++++ & +++ & +++ \\
IGFR-1 & ++++ & +++ & +++ \\
\hline
\end{tabular}

Staining was interpreted by pathologist visual scoring as strong $(++++$ and +++$)$, moderate $(++)$, and weak $(+)$ intensity. Five selected sections were analyzed per animal.

alcoholic rats presented smaller areas compared to controls. The average perimeter of the nuclei and cytoplasm of epithelial cells differed significantly between alcoholic and control groups at 90, 180 , and 270 days, similar to the morphometric analysis presented in our work. Conversely, the present work showed no significant difference in the plasma concentration of $\mathrm{FSH}, \mathrm{LH}$, progesterone, and $17 \beta$-estradiol. Gavaler, Van Thiel, and Lester (1980) noted in Wistar rats that uteri and oviducts exhibited atrophy of the epithelial lining with cuboidal cells and no signs of secretory activity. Hormone plasma assays revealed similar levels of LH and $17 \beta$-estradiol in alcoholic and control rats. However, progesterone was lower and FSH was higher following ethanol intake. Buthet et al. (2013) observed a significant decrease in the uterine horn weight following alcohol ingestion. According to Van Thiel, Gavaler, and Lester $(1977,1978)$, the epithelial layer of the uterine endometrium and uterine tubes was composed of cuboidal cells with no signs of secretory activity similar to ovariectomized rats. Also, alcoholic rats showed low levels of $17 \beta$-estradiol and progesterone compared to control and isocaloric animals. Decreased circulating sex hormones and alterations in behavioral and cognitive aspects of reproduction were observed by Dallman et al. (2002). Buthet et al. (2013) suggested that acetaldehyde accumulation and the oxidative stress could be additional factors contributing to the alcoholrelated hormonal changes, thus influencing the uterine tissue of rats.

Some findings related to the structure of the uterine epithelium of UCh rats were described by Martinez et al. (2001) and Buthet et al. (2013), who also observed dilation of the cisterns of rough endoplasmic reticulum, mitochondrial edema, accumulation of lipid droplets, intense vacuolization, and deformed nuclei in the uterine epithelial cells. According to Buthet et al. (2013), this intense disorganization of cellular structure suggests the action of a chemically induced cell injury. Decrease in cellular defenses such as a significant reduction in GSH content, an increase in the GSSG, and a decrease in the GSH/GSSG ratio may lead to oxidative stress, as

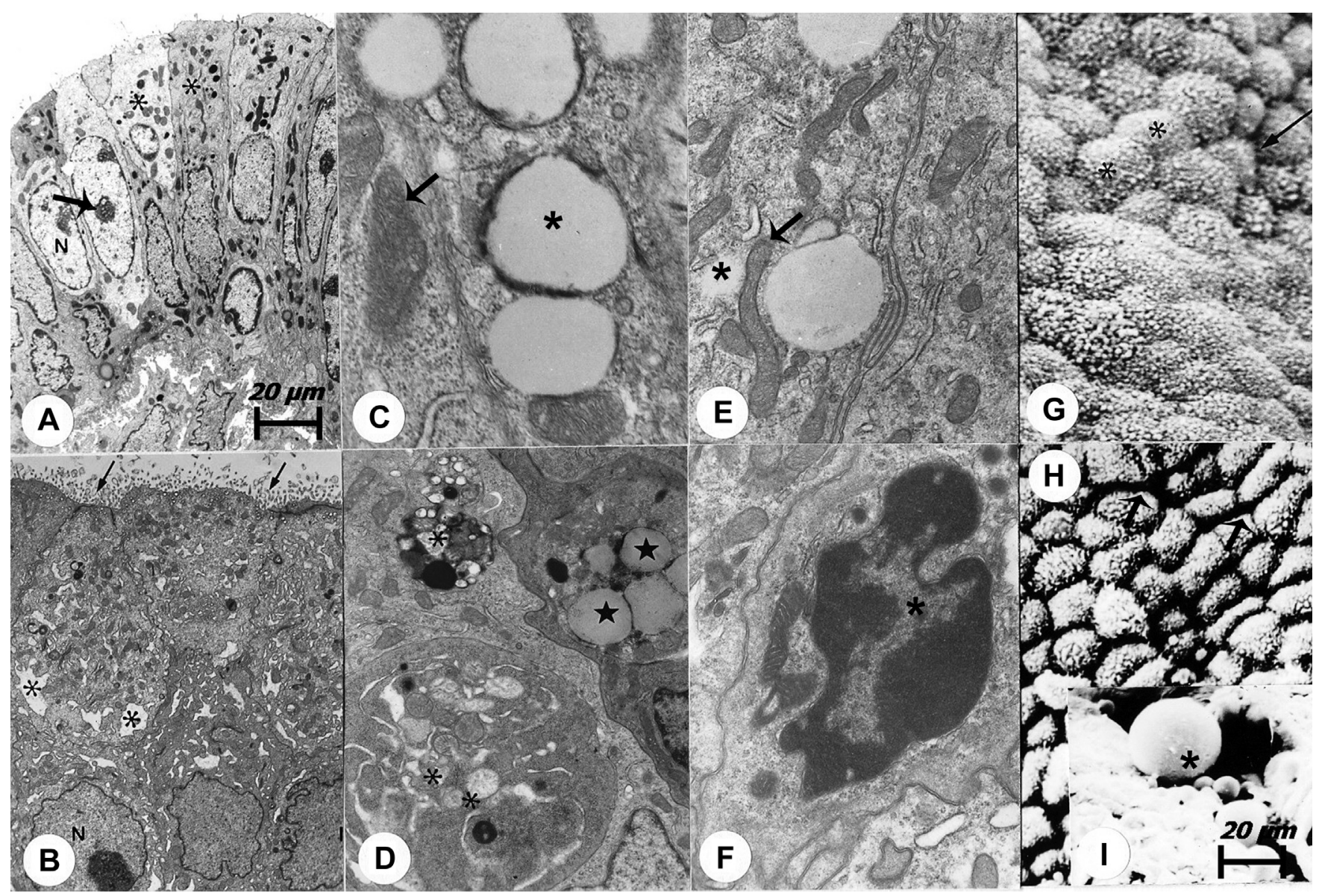

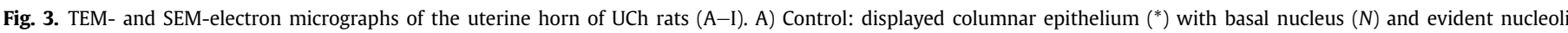

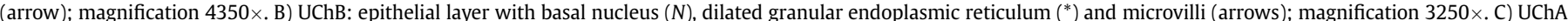

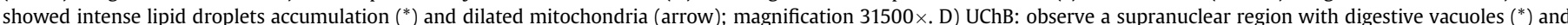

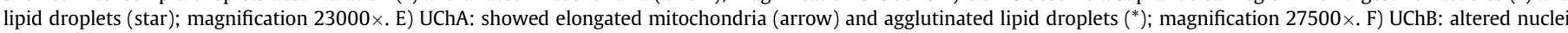

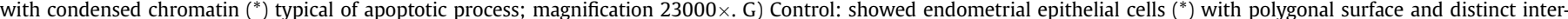

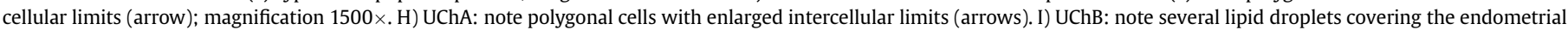
surface $\left({ }^{*}\right)$; magnification $800 \times$. 
already demonstrated by our group in female reproductive tissues of UCh rats (Chuffa et al., 2011). However, hormonal changes might also be involved in such structural and ultrastructural lesions. Hirata and Hirata (1991) showed that the increase in acetate alters fatty acid metabolism by inhibiting lipolysis, thereby contributing to the formation of hepatic steatosis. The accumulation of fatty acids promotes increased concentration of acetate in the liver, promoting its release into blood plasma, which can be degraded to obtain energy or even accumulated in the form of fatty acids and cholesterol in extra-hepatic tissues. Studies have suggested that the oxidizing agent produced during the metabolism of ethanol is able to disrupt membrane potential and mitochondrial permeability. The rat liver mitochondria exposed to ethanol have shown significant reduction in membrane potential after $30 \mathrm{~min}$ of administration, suggesting that mitochondrial depolarization is the initial event of cellular injury associated with ethanol exposure. In addition, the augmentation in mitochondrial membrane permeability related to the mega-opening of channels promotes rapid influx of water and ions and consequent mitochondrial edema (Adachi \& Ishii, 2002). The tissue injury has also been correlated with changes in the endoplasmic reticulum and Golgi complex. Studies have shown that ethanol alters the metabolism of amino acids, including decreased conversion of homocysteine to methionine, so that higher plasma homocysteine concentration has been observed as a result of chronic exposure to ethanol (Ji \& Kaplowitz, 2003).

Notably, we found increased levels of caspase-3, while $\mathrm{Bcl} 2$ and Xiap appeared to be moderate in UChA rats. Apoptosis may trigger the events related to tissue damage, such as reduction in cell volume, dilation of endoplasmic reticulum, changes in the plasma membrane, and discontinuous chromatin condensation around the nuclear periphery which occurs at the beginning of the cell death process (Wyllie, 1997). High levels of Ki-67 and IGFR-1 indicated that the regulation of cell growth and differentiation were normally maintained in the endometrium. The low-ethanol preference observed in the UChA strain has been linked to the polymorphism of the mitochondrial aldehyde dehydrogenase (ALDH2) gene, followed by low NADH oxidation, which can promote a slow metabolism of acetaldehyde and its subsequent elimination. Low levels of dopamine and its derived metabolites in the nucleus accumbens of UChB rats may be related to a genetic predisposition to ethanol consumption (Quintanilla, Bustamante, Tampier, Israel, \& HerreraMarschitz, 2007). Sapag et al. (2009) have reported large variations in amino acid sequences between UChA and UChB rats, suggesting that mitochondrial genes could be important factors associated with maternal genetic predisposition to alcoholism, which may lead us to conclude that the differences in the expression of these apoptotic factors is more evident in UChA rats.

In summary, chronic ethanol intake leads to structural and molecular alterations in the uterine endometrial cells of UCh rats, regardless of whether low doses or high-doses were consumed, promoting reproductive disorders.

\section{Acknowledgments}

This work was supported by a grant from Research Foundation of Sao Paulo State (FAPESP Procs. 03/11244-6, 04/03757-6).

\section{References}

Adachi, M., \& Ishii, H. (2002). Role of mitochondria in alcoholic liver injury. Free Radical Biology \&' Medicine, 32, 487-491.

Banzatto, A. D., \& Kronka, S. N. (1989). Experimentação agrícola. Jaboticabal, Brazil: Funep.

Bo, W. J., Kruegen, W. A., Rudeen, P. K., \& Symmes, S. K. (1982). Ethanol-induced alterations in the morphology and function of the rat ovary. The Anatomical Record, 202, 255-260.
Buthet, L. R., Maciel, M. E., Quintans, L. N., Rodríguez de Castro, C., Costantini, M. H. Fanelli, S. L., et al. (2013). Acetaldehyde content and oxidative stress in the deleterious effects of alcohol drinking on rat uterine horn. Journal of Toxicology, 161496. http://dx.doi.org/10.1155/2013/161496.

Chuffa, L. G., Amorim, J. P., Teixeira, G. R., Mendes, L. O., Fioruci, B. A., Pinheiro, P. F, et al. (2011). Long-term exogenous melatonin treatment modulates overall feed efficiency and protects ovarian tissue against injuries caused by ethanolinduced oxidative stress in adult UChB rats. Alcoholism: Clinical and Experimental Research, 35, 1498-1508.

Chuffa, L. G. Seiva, F. R., Fávaro, W. J., Amorim, J. P., Teixeira, G. R., Mendes, L. O., et al. (2013). Melatonin and ethanol intake exert opposite effects on circulating estradiol and progesterone and differentially regulate sex steroid receptors in the ovaries, oviducts, and uteri of adult rats. Reproductive Toxicology, 39, 40-49.

Chu, J., Tong, M., \& Monte, S. M. (2007). Chronic ethanol exposure causes mitochondrial dysfunction and oxidative stress in immature central nervous system neurons. Acta Neuropathologica, 113, 659-673.

Clave, S., Joya, X., Salat-Batlle, J., Garcia-Algar, O., \& Vall, O. (2014). Ethanol cytotoxic effect on trophoblast cells. Toxicology Letters, 225, 216-221.

Dallman, M. F., Viau, V. G., Bhatnagar, S., Gomez, F., Laugero, K., \& Bell, E. (2002). Corticotropin-releasing factor, corticosteroids, stress, and sugar: energy balance, the brain, and behavior. In Pfaff, D. W., Arnold, A. P., Etgen, A. M., Fahrbach, S. E. \& Rubin, R. T. (Eds.). Hormones, brain and behavior, Vol. 9 (pp. 571-632). San Diego, CA: Elsevier.

Day, N. L., Helsel, A., Sonon, K., \& Goldschmidt, L. (2013). The association between prenatal alcohol exposure and behavior at 22 years of age. Alcoholism: Clinical and Experimental Research, 37, 1171-1178.

Fernandez-Sola, J., Nicolas, J. M., Estruch, R., \& Urbano-Marquez, A. (2005). Gender differences in alcohol pathology. In Preedy, V. R., \& Watson, R. R. (Eds.) Comprehensive handbook of alcohol related pathology, Volume 1 (pp. 261-278). London: Elsevier Science Ltd - Academic Press, (2005).

Gavaler, J. S., Van Thiel, D. A., \& Lester, R. (1980). Ethanol: a gonadal toxin in the mature rat of both sexes. Alcoholism: Clinical and Experimental Research, 4, $271-276$.

Grinfeld, H., Goldenberg, S., Segre, C. A. M., \& Chadi, G. (1999). Effects of ethanol on offspring of C57BL/6J mice alcoholized during gestation. Acta Cirurgica Brasileira, 13, 101-107.

Hirata, E. S., \& Hirata, L. C. M. (1991). Bioquímica e metabolismo do ethanol. In J. R. A. Fortes, \& W. N. Cardoo (Eds.), Alcoolismo: diagnóstico e tratamento (pp. 57-63). São Paulo: Savier.

Hochgraf, P. B., Zilberman, M. L., \& Andrade, A. (1995). Women alcoholics-social, demographic and clinical characteristics in a Brazilian sample. Alcohol and Alcoholism, 30, 427-432.

Ji, C., \& Kaplowitz, N. (2003). Betaine decreases hyperhomocysteinemia, endoplasmic reticulum stress, and liver injury in alcohol-fed mice. Gastroenterology, $124,1488-1499$.

Kesmodel, U., Wisborg, K., Olsen, S. F., Henriksen, T. B., \& Secher, N. J. (2002) Moderate alcohol intake in pregnancy and the risk of spontaneous abortion. Alcohol and Alcoholism, 37, 87-92.

Lyngsø, J., Toft, G., Høyer, B. B., Guldbrandsen, K., Olsen, J., \& Ramlau-Hansen, C. H. (2014). Moderate alcohol intake and menstrual cycle characteristics. Human Reproduction, 29, 351-358.

Mardones, J., \& Segovia-Riquelme, N. (1983). Thirty-two years of selection of rats by ethanol preference: UChA and UChB strains. Neurobehavioral Toxicology and Teratology, 5, 171-178.

Martinez, M., Garcia, P. J., Cagnon, V. H., Mello Júnior, W., Padovani, C. R., \& Martinez, F. E. (2001). Effects of chronic experimental alcoholism on the epithelium of the uterine horn of rats (Rattus norvegicus albinus). Journal of Submicroscopic Cytology and Pathology, 33, 107-115.

Martinez, M., Martinez, F. E., Garcia, P. J., Cagnon, V. H., Mello Júnior, W., \& Padovani, C. R. (1999). Morphometric analysis of the endometrial epithelium of rats (Rattus norvegicus albinus) submitted to chronic experimental alcoholism. Journal of Submicroscopic Cytology and Pathology, 31, 469-475.

Oliveira, S. A., Chuffa, L. G., Fioruci-Fontanelli, B. A., Lizarte Neto, F. S. Novais, P. C., Tirapelli, L. F., et al. (2014a). Apoptosis of Purkinje and granular cells of the cerebellum following chronic ethanol intake. Cerebellum, 13, $728-738$.

Oliveira, S. A., Fontanelli, B. A., Stefanini, M. A., Chuffa, L. G., Teixeira, G. R. Lizarte, F. S., et al. (2014b). Interaction of maternal separation on the UCh rat cerebellum. Microscopy Research and Technique, 77, 44-51.

Ornoy, A., \& Ergaz, Z. (2010). Alcohol abuse in pregnant women: effects on the fetus and newborn, mode of action and maternal treatment. International Journal of Environmental Research and Public Health, 7, 364-379.

Quintanilla, M. E., Bustamante, D., Tampier, L., Israel, Y., \& Herrera-Marschitz, M. (2007). Dopamine release in the nucleus accumbens (shell) of two lines of rats selectively bred to prefer or avoid ethanol. European Journal of Pharmacology, $573,84-92$.

Sapag, A., González-Martínez, G., Lobos-González, L., Encina, G., Tampier, L. Israel, Y., et al. (2009). Polymorphisms in mitochondrial genes encoding complex I subunits are maternal factors of voluntary alcohol consumption in the rat. Pharmacogenetics and Genomics, 19, 528-537.

Surtel, A., Klepacz, R., \& Wysokińska-Miszczuk, J. (2014). Alcohol dependence syndrome-symptoms in the oral cavity. Postępy Higieny I Medycyny Doświadczalnej, $68,828-833$. 
Van Thiel, D. H., Gavaler, J. S., \& Lester, R. (1977). Ethanol: a gonadal toxin in the female. Drug and Alcohol Dependence, 2, 373-380.

Van Thiel, D. H., Gavaler, J. S., \& Lester, R. (1978). Alcohol-induced ovarian failure in the rat. The Journal of Clinical Investigation, 61, 624-632.

Wallock-Montelius, L. M., Villanueva, J. A., Chapin, R. E., Conley, A. J., Nguyen, H. P., Ames, B. N., et al. (2007). Chronic ethanol perturbs testicular folate metabolism and dietary folate deficiency reduces sex hormone levels in the Yucatan micropig. Biology of Reproduction, 76, 455-465.

World Health Organization. (2011). Global status report on alcohol and health. Geneva: WHO Press:1-85.

Wyllie, A. H. (1997). Apoptosis and carcinogenesis. European Journal of Cell Biology 73, 189-197. 